\title{
A Cubesat Asteroid Mission: Propulsion Trade-offs
}

\author{
Geoffrey A. Landis ${ }^{1}$, Steven R. Oleson ${ }^{2}$, Melissa L. McGuire ${ }^{2}$, Michael J. Bur ${ }^{2}$, Laura M. Burke ${ }^{2}$, James E. Fittje ${ }^{3}$, \\ Lisa L. Kohout ${ }^{2}$, James Fincannon ${ }^{2}$, Thomas W Packard ${ }^{3}$ and Michael C. Martini ${ }^{4}$ \\ NASA John Glenn Research Center, 21000 Brookpark Road, Cleveland, OH 44135
}

\begin{abstract}
A conceptual design was performed for a 6-U cubesat for a technology demonstration to be launched on the NASA Space Launch System (SLS) test launch EM-1, to be launched into a free-return translunar trajectory. The mission purpose was to demonstrate use of electric propulsion systems on a small satellite platform. The candidate objective chosen was a mission to visit a Near-Earth asteroid. Both asteroid fly-by and asteroid rendezvous missions were analyzed. Propulsion systems analyzed included cold-gas thruster systems, Hall and ion thrusters, incorporating either Xenon or Iodine propellant, and an electrospray thruster. The mission takes advantage of the ability of the SLS launch to place it into an initial trajectory of $\mathbf{C} 3=0$.
\end{abstract}

\section{Nomenclature}

$\begin{array}{ll}\text { C3 } & =\text { Hyperbolic excess, } \mathrm{km}^{2} / \mathrm{s}^{2} \\ \mathrm{EM}-1 & =\text { Initial Exploration Mission, the first launch of the SLS vehicle } \\ \mathrm{g} & =\text { Earth's gravitational constant, } 9.82 \mathrm{~m} / \mathrm{s}^{2} \\ \mathrm{Isp} & =\text { Specific impulse, seconds } \\ \mathrm{SLS} & =\text { The Space Launch System launch vehicle } \\ \mathrm{T}_{\mathrm{b}} & =\text { Duration of thrust (burn time), seconds } \\ \mathrm{U} & =\text { Cubesat unit. One unit is } 10 \mathrm{~cm} \text { by } 10 \mathrm{~cm} \text { by } 10 \mathrm{~cm} \\ \mathrm{~V}_{\mathrm{e}} & =\text { Exhaust velocity, } \mathrm{m} / \mathrm{s} \\ \alpha & =\text { Specific power of the electric power system, watts of exhaust energy per unit mass, } \mathrm{W} / \mathrm{kg} \\ \Delta \mathrm{V} & =\text { Velocity change, } \mathrm{km} / \mathrm{s} \\ \eta_{\mathrm{t}} & =\text { Energy efficiency of the thruster system, equal to kinetic energy of exhaust divided by energy input to } \\ & \text { the thruster }\end{array}$

\section{Introduction}

$\mathrm{C}$ UBESATS represent a class of small satellites that are designed to fit within a very low mass budget and tightly constrained volume ${ }^{1}$. To date, cubesat missions have only been operated in Earth orbit, typically as "ride along" secondary payloads on other missions, but there is considerable interest in expanding the applicability of cubesat type spacecraft into lightweight, low cost missions beyond Low Earth Orbit.

A conceptual design was performed for a 6-U cubesat for a technology demonstration to be launched on the NASA Space Launch System (SLS) test launch EM-1, to be launched into a free-return translunar trajectory. The mission purpose was to demonstrate use of electric propulsion systems on a small satellite platform. The candidate objective chosen was a mission to visit a Near-Earth asteroid. Both asteroid fly-by and asteroid rendezvous missions were analyzed.

Propulsion systems analyzed included cold-gas thruster systems, Hall and ion thrusters, incorporating either Xenon or Iodine propellant, and an electrospray thruster. The mission takes advantage of the ability of the SLS launch to place it into an initial trajectory of $\mathrm{C} 3=0$.

\footnotetext{
${ }^{1}$ Research Scientist, Photovoltaic and Electrochemical Systems Branch, NASA John Glenn Research Center, 21000 Brookpark Road, MS 302-1, Cleveland, OH 44135; AIAA Associate Fellow

${ }^{2}$ NASA John Glenn Research Center, 21000 Brookpark Road, Cleveland, OH 44135

${ }^{3}$ Vantage Partners, LLC, NASA Glenn Research Center, 21000 Brookpark Road, MS 142-6, Cleveland, OH 44135

${ }^{4}$ Zin Technologies, Inc., NASA Glenn Research Center, 21000 Brookpark Road, MS 105-3, Cleveland, OH 44135
} 


\section{Background}

The NASA Space Launch System (SLS) is a next-generation heavy-lift booster, intended to be the launch vehicle for future exploration missions ${ }^{2}$. The first launch of SLS is EM-1, "Initial Exploration Mission," a demonstration mission launched without crew into a free-return translunar trajectory. The launch is currently scheduled for December $2017^{3}$. The primary purpose of this launch is to demonstrate the booster and integrated

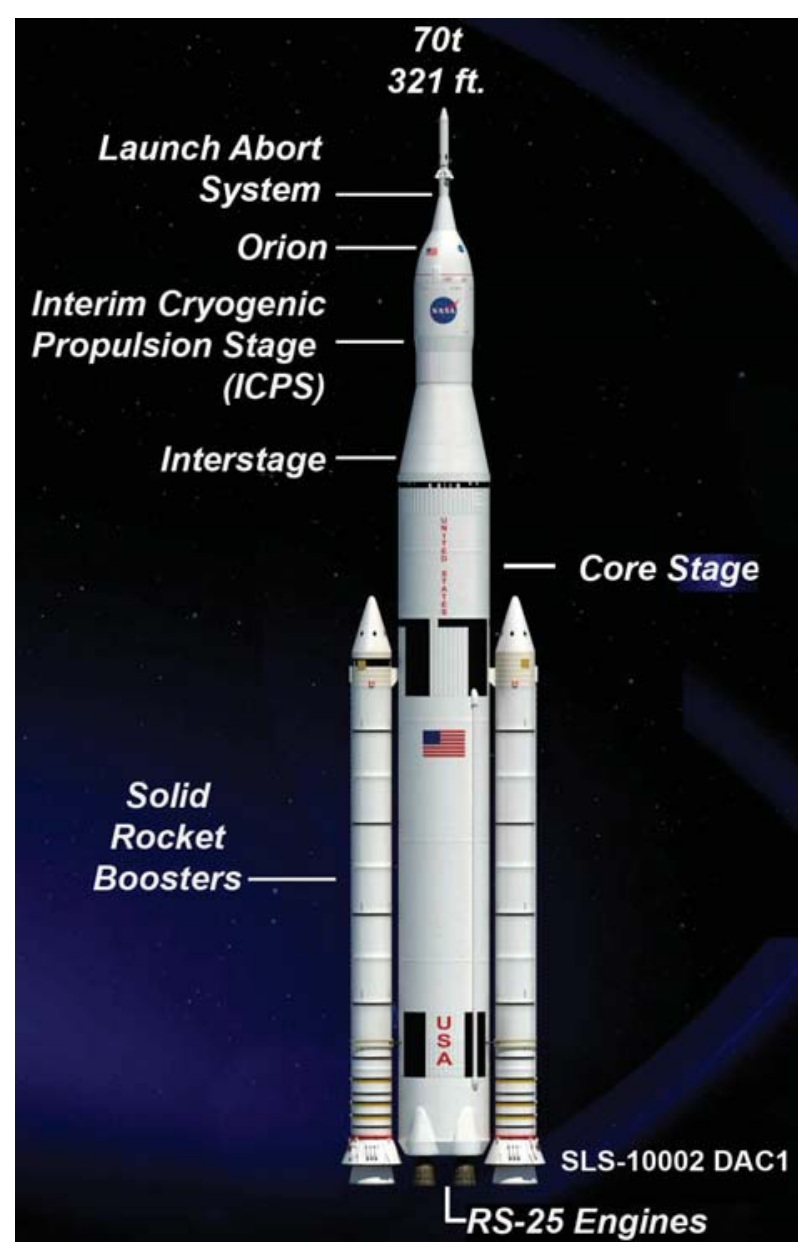

Figure 1. EM-1 launch configuration for the SLS spacecraft systems performance prior to a crew-carrying flight, including possibly demonstrating high-speed entry, at $11 \mathrm{~km} / \mathrm{sec}$, of the crew return capsule from a trans-lunar orbit. However, in addition to the primary missions, the vehicle has considerable payload excess capability, and is being planned to carry secondary payloads, and is projected to carry cubesats for deployment.

The Collaborative Modeling for Parametric Assessment of Space Systems ("COMPASS") team at NASA Glenn, a multidisciplinary concurrent engineering team that performs integrated conceptual design studies and trade studies for space missions, was given the task of designing a technology demonstration vehicle that could fly on the EM-1 mission, cubesat, with the constraints:

- Launch on EM-1 mission

- Fits within a cubesat envelope

- Demonstrate operation beyond low Earth orbit

- Demonstrate one (or more) technologies applicable to future missions.

In addition, the challenge was given that the mission should not merely operate the technologies to be demonstrated, but should demonstrate the use of the technology in the context of performing an operational mission: it is to go somewhere and accomplish a science mission.

The selected mission is to demonstrate the use of electric propulsion systems on a cubesat platform, performing a fly-by or rendezvous with a near-Earth object (NEO). The mission design was given the name "Diminutive Asteroid Visitor using Ion Drive," or "DAVID."4

The vehicle is shown in Figure 2. Conceptual design of the mission will be presented elsewhere. ${ }^{45}$ This work focuses on the propulsion system trade-off, looking at the advantages and disadvantages of different propulsion systems for the mission.

\section{A. Asteroid Mission}

The science objective takes advantage of the ability of the SLS to launch the cubesat into an escape orbit. Although the majority of asteroids are confined to orbits within the asteroid belt, there exist a number of near-Earth objects, including a significant fraction which are Earth crossing asteroids, some of which fly past the Earth with closest pass distances of a million kilometers or less. The concept here is to find such an Earth-flyby object, and use the low-thrust propulsion system to move the cubesat to a position near the closest-approach location. In essence, instead of the vehicle flying past the asteroid, we position the vehicle so that the asteroid flies by it.

Depending on which asteroid is chosen, the velocity of the asteroid relative to the Earth can range from less than $0.5 \mathrm{~km} / \mathrm{s}$ to over $40 \mathrm{~km} / \mathrm{s} .{ }^{6}$ For a good view of the asteroid during the fly-by, we limit our choices to asteroids with relative velocity of under $1.5 \mathrm{~km} / \mathrm{s}$, with lower velocities preferred.

The fact that some of these NEOs make close approaches at relative velocities under $5 \mathrm{~km} / \mathrm{sec}$ allows us to consider an even more ambitious goal: a rendezvous mission. 

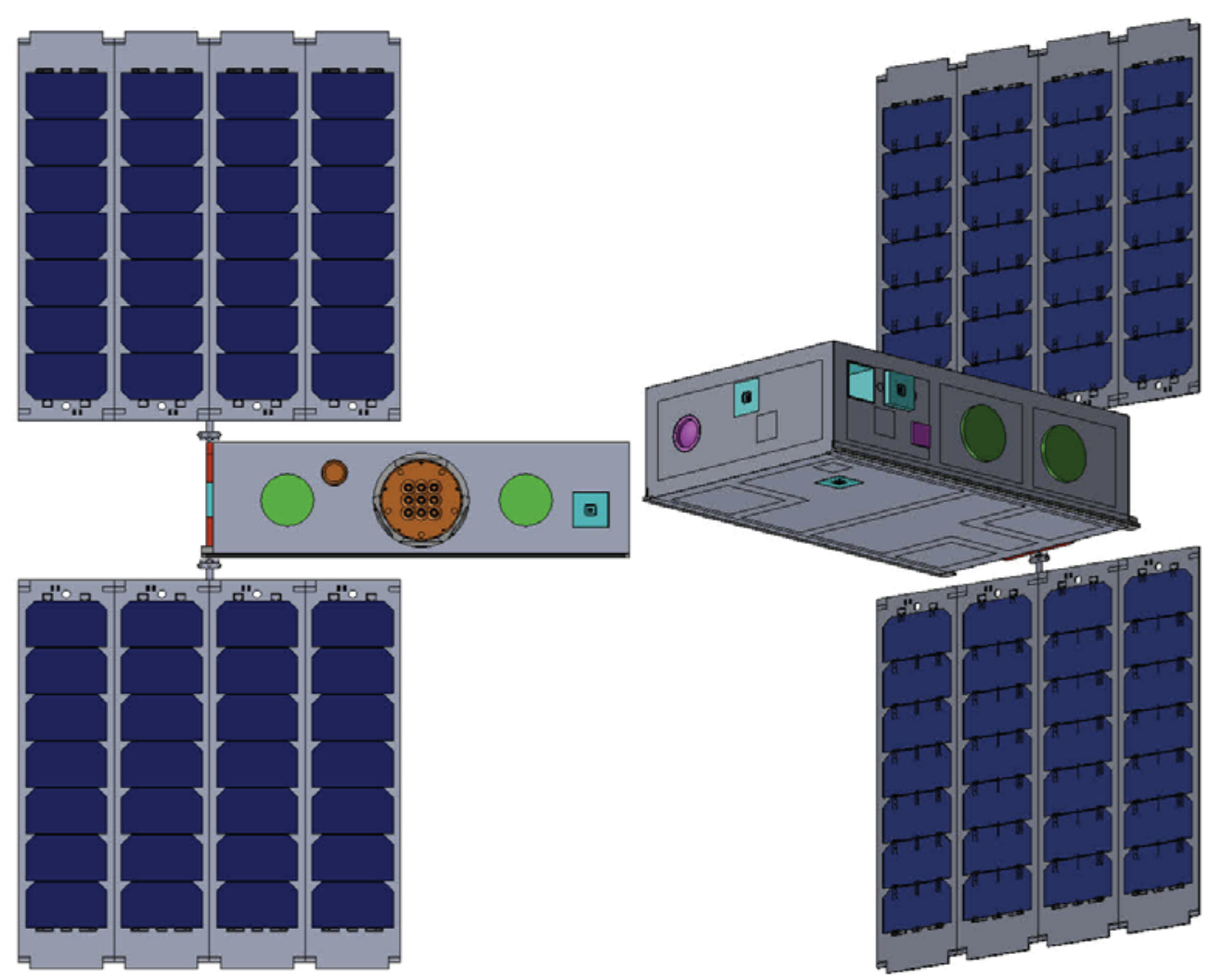

Figure 2. Design views of $6 \mathrm{U}$ electric-propulsion cubesat

\section{B. SLS Launch and Trans-Lunar Trajectory}

The launch of EM-1 (nominal date: December 17, 2017) places the vehicle into Earth orbit, and the upper stage does a trans-lunar injection (TLI) burn, which places the vehicle onto a free-return trajectory designed to take it around the moon and back to the Earth. At about $30 \mathrm{~min}$ after the TLI burn, the upper stage then separates from the crew vehicle and makes a disposal burn of about $15 \mathrm{~m} / \mathrm{s}$ to put it on a lunar flyby trajectory which will give it a gravitational slingshot to Earth escape. ${ }^{3}$

There are thus four possible points in the mission at which the DAVID cubesat can be separated from the vehicle, resulting in four different possible initial trajectories.

Separating the cubesat after the EM-1 achieves its initial Earth orbit but prior to the TLI burn places the cubesat into Earth orbit. Ejecting into this starting orbit does not take advantage of the SLS's translunar trajectory, and would require onboard propulsion onboard to achieve escape. This option was beyond the capability of the mission, and not considered.

Keeping the cubesat with the upper stage would put it into an escape trajectory, with a hyperbolic excess C 3 of $\sim 1.5 \mathrm{~km}^{2} / \mathrm{s}^{2}$. Since the cubesat is a secondary payload on the mission, the direction of the escape trajectory cannot be assumed to be in the optimum direction for an asteroid mission. The vector is determined by the swing past the moon, and will depend on the position of the moon on the date of the launch. Since the launch date is not under our control, we cannot count on a launch onto any particular escape vector. The possibilities are that (1) the launch is in the "right" direction for our chosen asteroid, contributing part of the $\Delta \mathrm{V}$ required to reach the asteroid; (2) the launch is in the "wrong" direction, increasing the $\Delta \mathrm{V}$ required to reach the asteroid; (3) the launch is in an irrelevant direction for our chosen asteroid (i.e., neither increasing nor decreasing the propulsion requirement.) Since we do not know this in advance (and it may change with a small slip in launch date), we set this option aside to be reconsidered if future information becomes available.

However, a small trajectory correction on the outbound leg will allow us to modify the lunar swingby to put us in a lunar fly-by trajectory with $\mathrm{C} 3$ of 0 . The baseline analysis here assumes the mission is ejected from the upper stage with such a correction maneuver following ejection, so that the cubesat is in an initial trajectory which has escaped from Earth, but with zero (or negligible) velocity relative to Earth. 
Alternative options are to separate the cubesat on the free-return trajectory, either during the outbound coast, or the inbound coast. On the outbound leg, the on-board EP system again adjusts the lunar fly-by trajectory to bring the excess velocity $\mathrm{C} 3$ to 0 . If the vehicle continues on the free-return trajectory, it could make a propulsive maneuver on the inbound leg of the trajectory to avoid hitting the Earth. Since the return trajectory is intended to have atmospheric entry at nearly escape velocity, only a small propulsive maneuver is required to reach a $\mathrm{C} 3$ of 0 . These options achieves essentially the same result as the previous separation, which is to put the cubesat at a C 3 of zero (i.e., co-orbital with the Earth).

\section{Choice of Target}

A total of 12874 close approaches by asteroids were analyzed, with the list narrowed down to objects making nearest approach no earlier than 2019 (at least 1 year after the launch date), but no later than 2020*. Several candidate objects were selected. Of these candidates, the object 2001 GP2 was chosen as a target. This object makes a close approach to the moon at 3.2 times lunar distance (1.2 million kilometers) in October 2020, with a relatively slow velocity relative to Earth of $2.35 \mathrm{~km} / \mathrm{s}$. The size, type and composition of this asteroid is not known, however, from its visual magnitude of 26.3 , if we assume a typical asteroid albedo of 0.15 , we can estimate that it is an 18 meter diameter asteroid.

Targeting asteroids that fly close to Earth minimizes the propulsion required for fly-by or rendezvous, but does not eliminate it. The $\Delta \mathrm{V}$ required is still significant. Calculations using a low-thrust trajectory $\operatorname{code}^{7,8}$ show that from a C3 of zero, it requires a total $\Delta \mathrm{V}$ of approximately $400 \mathrm{~m} / \mathrm{s}$ to accomplish the fly-by of $2001 \mathrm{GP} 2$ (2020 flyby).

A larger $\Delta \mathrm{V}, \sim 2000 \mathrm{~m} / \mathrm{s}$, is required to achieve a rendezvous. Once rendezvous is achieved, the spacecraft is coorbital with the target. The gravity of $2001 \mathrm{GP}$ is small enough that the spacecraft flies in formation with it, rather than orbiting the body. The rendezvous requires a flight time of about 1000 days, of which about half, 500 days, is spent thrusting.

2001 GP2 was just chosen as an example. In the event of a change in schedule, many other asteroid fly-bys opportunities exist, although not all have such low relative velocity to Earth.

In the event that the $\Delta \mathrm{V}$ from the SLS injection is in (or near) the desired trajectory direction, as discussed in the previous section, it can reduce the $\Delta \mathrm{V}$ required for asteroid flyby and rendezvous. This was not assumed in the analysis. In the best case, if a spacecraft is designed for the rendezvous mission, a favorable $\Delta \mathrm{V}$ from the SLS launch can allow a fly-by mission plan to be changed to a rendezvous.

\section{Mission}

\section{A. Conops}

The strawman Concept for Operations (conops) for the asteroid rendezvous mission is as follows:

1. Install DAVID powered down on dispenser

2. Launch

3. Deploy from upper stage after MPCV separation

- $\quad$ Spring switch to 'turn-on' spacecraft

- Assume sufficient separation from upper stage

- Assume that the stage avoids the (potential) "cloud" of other cubesats sharing the ride

4. De-tumble by spinning up wheels, acquires sun pointing and attitude, deploys solar arrays $(\sim 10$ minutes)

5. Communicates with ground to acquire location

6. Early demonstration opportunity: perform imaging inspection of spent Cryo Propulsion Stage (practice asteroid imaging)

7. Checkout/Initial Orbit Determination

8. Trajectory correction burn, if needed, to place vehicle on initial trajectory at $\mathrm{C} 3=0$

\footnotetext{
* If the criteria are slightly relaxed, object 2013 PA7 makes a close approach at a somewhat farther distance, 35 times lunar distance (14 million $\mathrm{km}$ ), at an extremely show relative velocity of $0.5 \mathrm{~km} / \mathrm{s}$. However, this approach in November 21, 2018 is slightly outside our desired window of one year after the EM-1 launch.
} 
9. Begins thrusting

- $\quad$ Stop thrust to coast every 1 week (deep space) to acquire new thrust pointing from ground tracking

- 12 hrs duration coast

- Low thrust to asteroid rendezvous ( 1000 days)

- Perform 'drift' science (space weather) and Tech demos during thrust mission

10. Final approach

- Ground based radars locate position of asteroid to direct DAVID to asteroid

- DAVID camera will be used to acquire asteroid position relative to spacecraft for final approach

- Co-orbit

11. Science 3 months: imaging 3 Megapixel images, asteroid gravity science, $\sim 300$ bps data return

- Max 3 year mission

12. Decommission

\section{IV: Propulsion}

The largest cubesat which can fit into a standard deployer is a 6-unit ("6-U") cubesat, which has dimensions of $10 \mathrm{~cm}$ by $20 \mathrm{~cm} \times 30 \mathrm{~cm}$ in its undeployed state. The allowable mass is $\sim 2 \mathrm{~kg}$ per unit, allowing a maximum mass for the cubesat of $12 \mathrm{~kg}$. This puts severe constraints on the amount of propellant that can be carried, and hence $\Delta \mathrm{V}$ that can be achieved, and on the area of solar arrays that can be deployed, and hence on the power available. Since the impulse per unit energy is inversely proportional to specific impulse, high specific impulse systems require large power systems. Hence, there is an optimum specific impulse for the mission, where higher specific impulse than optimum means that the power system dominates the mass, while lower specific impulse than optimum means that the propellant dominates the mass. For an ideal system in which the efficiency is independent of the specific impulse, the optimum specific impulse (or, equivalently, exhaust velocity) is: ${ }^{9,10}$

$$
V_{e}=g I_{s p}=\sqrt{2 \alpha \eta_{t} T_{b}}
$$

where $\alpha$ is the specific power of the electric power system, including power processing and the thermal systems, as well as all parts of the propulsion system that have mass proportional to the power; $\eta_{t}$ is the energy efficiency of the thruster; and $\mathrm{T}_{\mathrm{b}}$ is the duration of the thrust. At this exhaust velocity, the mass of the power system is equal to the propellant mass. Although this rule of thumb is only of approximate usefulness, since the burn time is a function of thrust, which itself depends on power and specific impulse, and hence does not substitute for numerical optimization, it is a useful rule for quick estimates of which types of propulsion system might be most suitable for a mission.

A number of choices for propulsion system were considered. In order to achieve sufficient $\Delta \mathrm{V}$ in the small constraints of a cubesat, specific impulse is at a premium. A summary of the types of propulsion systems available for cubesats can be found in Mueller, Hofer and Ziemer 2010, ${ }^{11}$ while a summary of design techniques for electric propulsion mission optimization can be found in Dankanich 2010. ${ }^{7}$

The propulsion choice interacts strongly with the vehicle design. Higher specific impulse systems require higher power. Control of tip-off, removing the initial rotational velocity from the deployment, can be a significant challenge. Depending on whether the thrust can be vectored, control of torque due to solar pressure on the arrays and due to thrust offset from the centerline may be a difficulty.

Cubesat propulsion systems are inherently less efficient that their larger satellite counterparts due to their small size. About 30\% propulsion efficiency for 10-100 Watt devices seem the best available, although work is ongoing on higher efficiency thrusters. This puts significant constraints on the power system.

In addition to mass, propellant volume constraints turned out to be critical: the 6-U platform is severely limited in its ability to carry large tanks of propellant, and hence higher density propellants were required in order to fit within the allowable volume.

Of the propulsion and power concepts analyzed, the final choices were narrowed down and more detailed cases run for Hall-effect thrusters (also called "Stationary Plasma Thruster", or SPT), small RF ion thrusters, and colloid electrospray thrusters. Six combinations of thruster, power system, and attitude control were run with detailed design scenarios; these are shown in Table 1. The Hall thruster has the greatest base of experience in operation in space, but the cases analyzed exceeded the initial mass allowance of $12 \mathrm{~kg}$, as did the Xe ion thruster. A case with two 10-W PUC (Propulsion Unit for Cubesats) ${ }^{12}$ electrospray thrusters also exceeded the mass allowance, but a revised case where this was reduced to lower power and a single thruster was run, which met the requirements.

Two cases were carried on to the final design. For the flyby mission, with a required $\Delta \mathrm{V}$ of approximately 400 $\mathrm{m} / \mathrm{s}$, the 10-Watt PUC electrospray thruster ${ }^{12}$ at a specific impulse of 1500 seconds was baselined (case 3.5 in table 1), incorporating a propellant-less cathode and a bellows salt tank. 


\begin{tabular}{|c|c|c|c|c|c|c|c|c|}
\hline Case & Power & Thruster & $\begin{array}{l}\text { Prop } \\
\text { load } \\
(\mathrm{kg})\end{array}$ & $\begin{array}{l}\Delta \mathrm{V} \\
(\mathrm{m} / \mathrm{s})\end{array}$ & $\begin{array}{l}\text { Thrust Time / } \\
\text { Trip Time }\end{array}$ & $\begin{array}{l}\text { Tip off } \\
\text { control }\end{array}$ & $\begin{array}{l}\text { Solar } \\
\text { Pressure/Thrust } \\
\text { Offset }\end{array}$ & $\begin{array}{l}\text { Initial } \\
\text { mass } \\
(\mathrm{kg})\end{array}$ \\
\hline 1 & $\begin{array}{l}160 \mathrm{~W} \\
\text { Articulated } \\
\text { Arrays }\end{array}$ & $\begin{array}{l}\text { BHT- } \\
200 \\
\text { (run at } \\
100 \mathrm{~W})\end{array}$ & $2.1^{*}$ & & $? / \sim 1000 \mathrm{~d}$ & Cold Gas & $\begin{array}{l}\text { Cold } \\
\text { Gas/wheels } \\
\text { (additional Xe } \\
\text { needed) }\end{array}$ & 19.5 \\
\hline 2 & $\begin{array}{l}30 \mathrm{~W} \\
\text { Articulated } \\
\text { Arrays }\end{array}$ & $\begin{array}{l}10-\mathrm{W} \\
1800 \mathrm{~s} \\
\text { Xe RF } \\
\text { Ion }\end{array}$ & 1.5 & $\sim 1300$ & $\sim 450 \mathrm{~d} / 1100 \mathrm{~d}$ & Cold Gas & $\begin{array}{l}\text { Cold gas plus } \\
\text { CG shift with } \\
\text { adjustable } \\
\text { mass }\end{array}$ & 12.7 \\
\hline 3 & $\begin{array}{l}42 \mathrm{~W} \\
\text { Articulated } \\
\text { Arrays }\end{array}$ & $\begin{array}{l}\text { Two } \\
1500 \mathrm{~s} \\
10-\mathrm{W} \\
\text { Colloid }\end{array}$ & 1.2 & $\sim 1250$ & $\sim 200 \mathrm{~d} / \sim 1200 \mathrm{~d}$ & $\begin{array}{l}\text { Reaction } \\
\text { wheels/thrust } \\
\text { gimbals }\end{array}$ & $\begin{array}{l}\text { Thruster } \\
\text { Gimbals }\end{array}$ & 13.7 \\
\hline 3.5 & $\begin{array}{l}30 \mathrm{~W} \\
\text { Articulated } \\
\text { Arrays }\end{array}$ & $\begin{array}{l}\text { One } \\
1500 \mathrm{~s} \\
10-\mathrm{W} \\
\text { Colloid }\end{array}$ & 1.1 & $\sim 1250$ & $\sim 200 \mathrm{~d} / \sim 1200 \mathrm{~d}$ & $\begin{array}{l}\text { Reaction } \\
\text { wheels/thrust } \\
\text { gimbals }\end{array}$ & $\begin{array}{l}\text { Thruster } \\
\text { Gimbals (solar } \\
\text { arrays for roll) }\end{array}$ & 11.5 \\
\hline 4 & $\begin{array}{l}\text { Non- } \\
\text { tracking, } \\
30 \mathrm{~W} \\
\text { Effective }\end{array}$ & $\begin{array}{l}\text { One } \\
1500 \mathrm{~s} \\
10-\mathrm{W} \\
\text { Colloid }\end{array}$ & 1 & $\sim 1250$ & $\sim 200 \mathrm{~d} / \sim 1200 \mathrm{~d}$ & Cold Gas & Spin-Stabilized & 11.5 \\
\hline 5 & $\begin{array}{l}\sim 100 \mathrm{~W} \\
\text { articulated }\end{array}$ & $\begin{array}{l}50-\mathrm{W} \\
2400 \mathrm{~s} \\
\text { Iodine } \\
\text { RF ion }\end{array}$ & 1.11 & 2088 & $\begin{array}{l}456 \mathrm{~d} / 611 \mathrm{~d} \\
\text { (rendezvous } \\
\text { mission) }\end{array}$ & $\begin{array}{l}\text { Reaction } \\
\text { wheels }\end{array}$ & $\begin{array}{l}\text { Thruster } \\
\text { Gimbal }\end{array}$ & 12.0 \\
\hline
\end{tabular}

Table 1: Summary trade-off chart of design studies for six combinations of thruster, power system, and attitude control

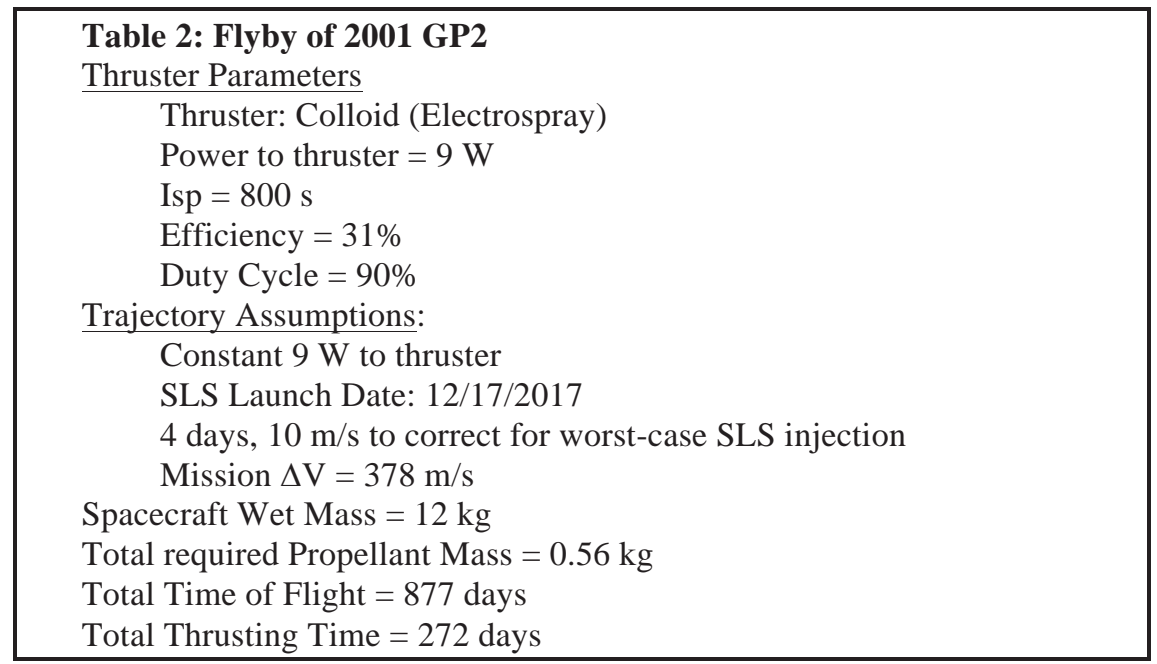




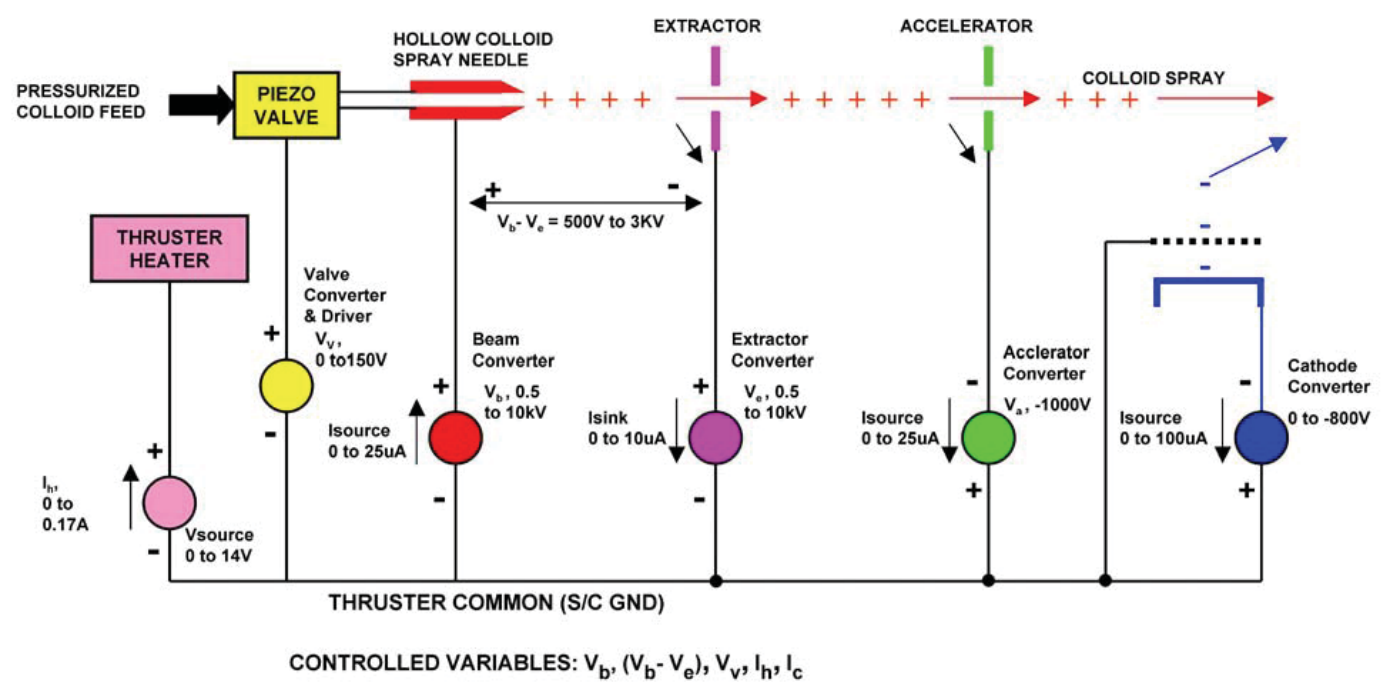

Figure 3. Block diagram of the colloid electrospray thruster

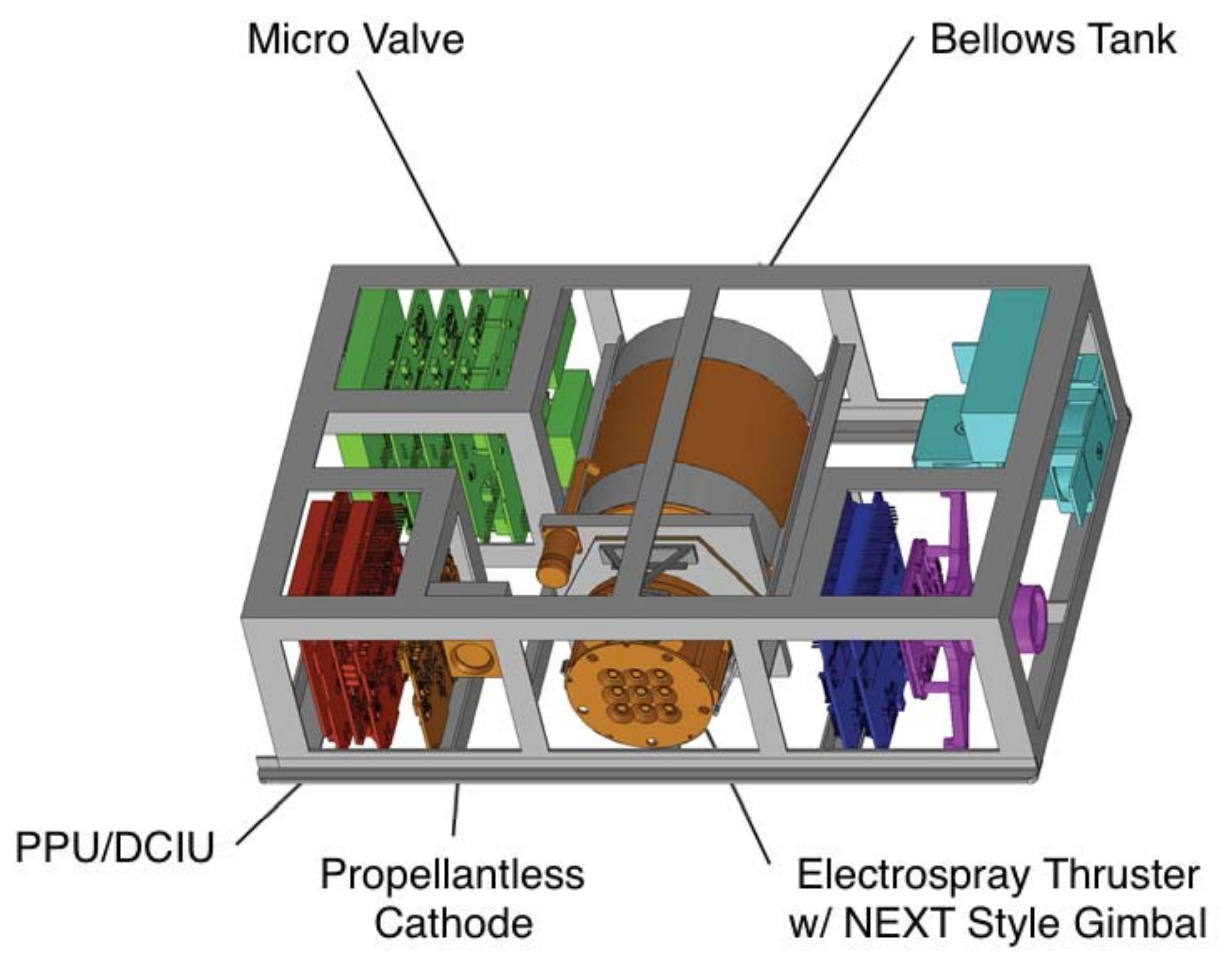

Figure 4. Interior layout of the cubesat with the electrospray thruster

Figure 3 shows a block diagram of the colloid electrospray thruster.

Due to volume constraints discussed, the high density of the electrospray salt was of great importance to the ability to carry sufficient propellant to achieve the required $\Delta \mathrm{V}$. The PUC thruster has a technology readiness level (TRL) between 4 and 5, due to development activities for the ST7 technology demonstration mission to demonstrate technologies for the LISA mission, ${ }^{12}$ and is planned for demonstration on 2015 LEO and 2016 GEO DARPA flights.

For the fly-by mission, the parameters are shown in table 2. In the analysis, margin is added at the system level on the SLS Cubesat Bus to carry a total of $30 \%$ growth on dry mass per each element. An additional $11 \%$ margin is carried on SLS Cubesat Bus (0.8 kg). 
Both spin-stabilized (2 RPM spin rate) and three-axis stabilized versions of the electrospray propulsion concept were analyzed. Both variants came in at about the same mass, $11.5 \mathrm{~kg}$. While the spin-stabilized version simplified the attitude control system, the thruster gimbal plus 3-Axis reaction wheels provides a more benign cubesat for secondary launch and more mission flexibility. It also allowed the use of approximately three times smaller solar arrays to achieve the same power level.

For the fly-by mission, the parameters are shown in table 2. Trajectory analysis was done with MALTO, "Mission Analysis Low-Thrust Optimization," a tool for low-thrust trajectory analysis and optimization, with relatively fast convergence for missions with gravity assists. ${ }^{8,13}$

For the more challenging rendezvous mission, with a $\Delta \mathrm{V}$ of approximately $2000 \mathrm{~m} / \mathrm{s}$, a higher specific impulse was needed. A 50-W RF-ion propulsion system was baselined. Although ion propulsion systems using Xenon propellant are well developed for use in space, because of the size of Xenon tanks required, Xenon propellant was ruled out. A higher density propellant, Iodine ${ }^{14}$, was baselined. Iodine has a considerably higher density, 4.9 grams $/ \mathrm{cm}^{3}$ (compared to $1.6 \mathrm{~g} / \mathrm{cm}^{3}$ for supercritical Xe), and can be stored at low pressure without cooling, allowing the propellant tanks to be lighter. Use of iodine in Hall-effect thrusters has been demonstrated. However, the use of iodine reduces the technology readiness to a lower TRL of 3-4, and this mission will require technology development and qualification. The mission also requires that the small ion thruster can be run for a total thrust time of 10,000 hours, which may require additional testing and verification.

The trajectory design assumptions are shown in table 3.

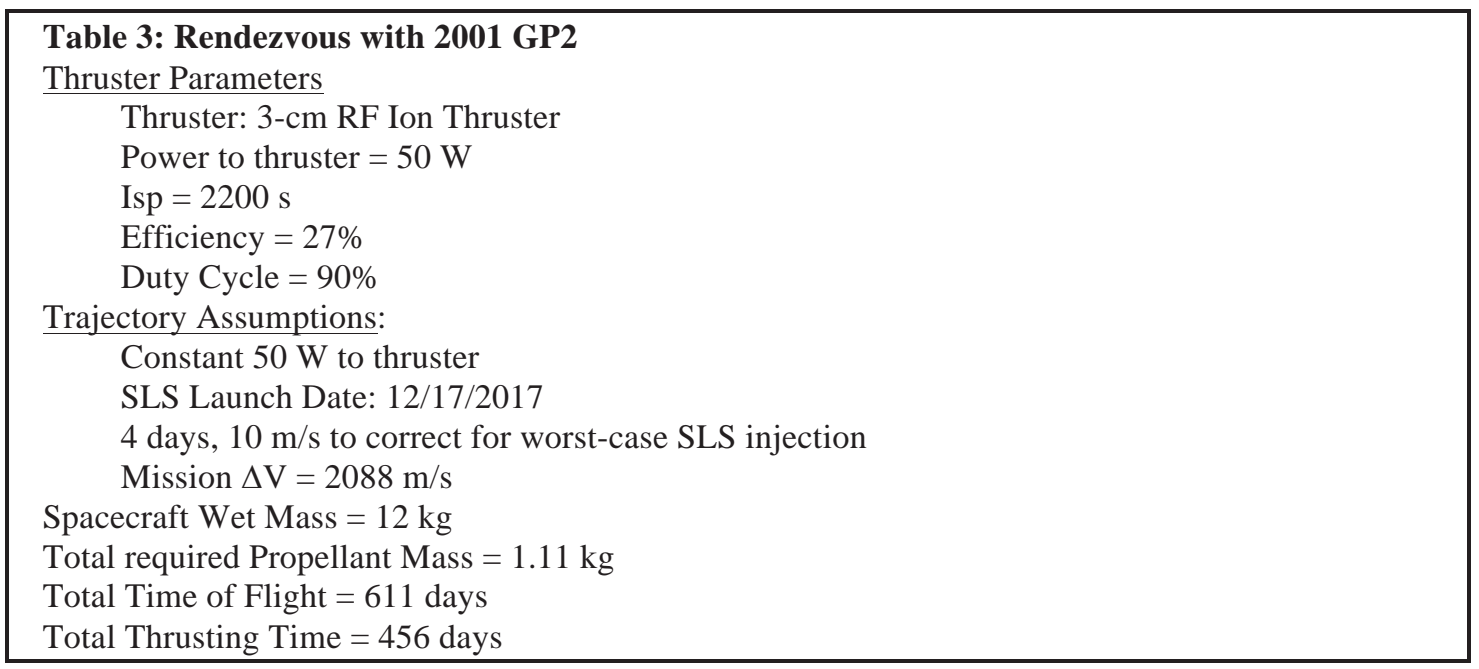

\section{Conclusions}

An asteroid mission is possible using a cubesat platform with high-efficiency electric propulsion.

Targeting asteroids that fly close to earth minimizes the propulsion required for flyby/rendezvous.

- The $\Delta \mathrm{V}$ required is still significant for a $6 \mathrm{U}$ cubesat: $\sim 400 \mathrm{~m} / \mathrm{s}$ flyby, $\sim 2000 \mathrm{~m} / \mathrm{s}$ rendezvous for $2001 \mathrm{GP} 2$ (2020 flyby)

- Many flybys opportunities exist but not all have such low relative velocity to earth.

- EM-1 places the vehicle on a trajectory C3 of $0.2 \mathrm{~km}^{2} / \mathrm{s}^{2}$ (using a $15 \mathrm{~m} / \mathrm{s}$ burn and a lunar flyby)

- Since the cubesat is a secondary payload on the mission, the direction of the escape trajectory cannot be assumed to be in the optimum direction for an asteroid mission

- Thus, the analysis here assumed the mission is ejected from the carrier during the translunar coast, and that the on-board EP system adjusts the lunar fly-by trajectory to take the excess velocity (C3) down to 0.

- In the event that the $\Delta \mathrm{V}$ from the SLS injection is in (or near) the desired trajectory direction, it can reduce the above $\Delta \mathrm{Vs}$ for asteroid flyby and rendezvous. This was not assumed in the analysis.

- Cubesat propulsion systems are inherently less efficient that their larger satellite counterparts due to their small size. About $30 \%$ propulsion efficiency for 10-100 W devices seem the best available, although work is ongoing on higher efficiency $(70 \%)$ electrospray thrusters.

- Due to mass constraints, high specific impulse is required for this mission.

- Due to volume constraints, the propellant density was also of great importance to the ability to achieve the required $\Delta \mathrm{V}$. This improves the relative usefulness of the electrospray salt, with higher propellant density. 
- In order to minimize high pressure tanks and volatiles, the salt electrospray and iodine ion propulsion systems were the optimum designs for the flyby and rendezvous missions respectively combined with a thruster gimbal and wheel system.

- For the candidate flyby mission, with a mission $\Delta \mathrm{V}$ of about $400 \mathrm{~m} / \mathrm{s}$, the mission objectives could be accomplished with a 10 Watt PUC electrospray propulsion system, at a specific impulse of 800s, incorporating a propellant-less cathode and a bellows salt tank. This propulsion system is planned for demonstration on 2015 LEO and 2016 GEO DARPA flights.

- For the rendezvous mission, at a $\Delta \mathrm{V}$ of $2000 \mathrm{~m} / \mathrm{s}$, the mission could be accomplished with a $50-\mathrm{W}$ miniature ion propulsion system running iodine propellant. This propellant system is not yet demonstrated in space.

\section{Acknowledgments}

We would like to acknowledge the work of the many members of the NASA Glenn COMPASS team who contributed to this study. The support of NASA GRC management, including Mr. Bryan Smith, Mr. Joel Kearns, Dr. John Sankovic, and Mr. Eli Naffah is acknowledged.

\section{References}

${ }^{1}$ Twiggs, B, "Origin of Cubesat," in Helvajian and Janson (eds.), Small Satellites: Past, Present, and Future, Chapter 5, AIAA: The Aerospace Press, 2008.

${ }^{2}$ May, T. A. and Creech, S. D., "NASA's Space Launch System: One Vehicle, Many Destinations," paper IAC13,A5.4-D2.8,2x17626, 64 $4^{\text {th }}$ International Astronautical Congress (IAC), Beijing China, 23-27 Sept. 2013.

3 Lyles, G. M., et al., "NASA Space Launch System (SLS) Development: Challenges and Solutions," $49^{\text {th }}$ AIAA/ASME/SAE/ASEE Joint Propulsion Conference, San Jose, CA, 15-17 Jul. 2013.

${ }^{4}$ Landis, G.A., Hepp, A. F., Stegeman, J.D., Oleson, S.R., Chandrashekhar, M.V.S., and the COMPASS Team, "A 6U-CubeSat Mission Analysis of a Near Earth Asteroid Visit," paper SC14-XI-7, 28th Annual AIAA/Utah State University Conference on Small Satellites, Logan, UT, 2-7 August 2014.

5 Landis, G. A., et al., "A Cubesat Asteroid Mission: Design Study and Trade-offs," to be presented, 65th International Astronautical Congress, Toronto, ON, 30 September- 2 October, 2014.

6 NASA Near Earth Object Program, NEO Earth Close-Approaches Between 1900 A.D. and 2200 A.D. http://neo.jpl.nasa.gov/cgi-bin/neo_ca

7 Dankanich, J. W., "Low Thrust Mission Design and Analysis," paper AIAA 2010-6857, $46^{\text {th }}$ AIAA/ASME/SAE/ASEE Joint Propulsion Conference and Exhibit, Nashville, TN, 25-28 July 2010.

${ }^{8}$ Kowalkowski, T., Rinderle, E., and Landau, D., Mission-Analysis Low-Thrust Optimization (MALTO) Users' Manual, California Institute of Technology, 2008..

${ }^{9}$ Stulinger, E., Ion Propulsion for Spaceflight, McGraw Hill, New York, 1964.

${ }^{10}$ Sutton, G. P., and Biblarz, O., "Electric Propulsion," Rocket Propulsion Elements, $7^{\text {th }}$ Edition, Chapter 19, pp. 660-710, John Wiley and Sons, 2001.

${ }^{11}$ Mueller, J., Hofer, R., and Ziemer, J., Survey of Propulsion Technologies Applicable to Cubesats, Jet Propulsion Laboratory, National Aeronautics and Space Administration, May 2010.

http://trs-new.jpl.nasa.gov/dspace/handle/2014/41627

${ }^{12}$ Randolph, T. M. et al., "Microthruster Propulsion for the Space Technology 7 (ST7) Technology Demonstration Mission," paper AIAA 2006-4320, 42 ${ }^{\text {nd }}$ AIAA/ASME/SAE/ASEE Joint Propulsion Conference and Exhibit, Sacramento, CA, 9-12 July 2006.

${ }^{13}$ Sims, J. A., Finlayson, P. A., Rinderle, E. A., Vavrina, M. A., and Kowalkowski, T. D., "Implementation of a Low-Thrust Trajectory Optimization Algorithm for Preliminary Design," paper AIAA 2006-6746, AIAA/AAS Astrodynamics Specialist Conference and Exhibit, Keystone, CO, 21-24 August 2006.

${ }^{14}$ Dankanich, J. W., Kamhawi, H., Szabo, J. and Pote, B., "Iodine Electric Propulsion for Interplanetary Small Satellite Application,” 1st Interplanetary Small Satellite Conference, Pasadena, CA, 20-21 June, 2013. 